Bryn Mawr College

Scholarship, Research, and Creative Work at Bryn Mawr College

Hermann Sauppe Library

Bryn Mawr College Publications, Special

Collections, Digitized Books

1874

\title{
Über die Anachronismen in den platonischen Gesprächen
}

Eduard Zeller

Let us know how access to this document benefits you.

Follow this and additional works at: http://repository.brynmawr.edu/digitizedbooks

Part of the Ancient Philosophy Commons

\section{Custom Citation}

Zeller, Eduard. 1874. Über die Anachronismen in den platonischen Gesprächen. Berlin: Konigl. Akademie der Wissenschaften (G. Vogt).

This paper is posted at Scholarship, Research, and Creative Work at Bryn Mawr College. http://repository.brynmawr.edu/digitizedbooks/32

For more information, please contact repository@brynmawr.edu. 
Über

\section{die Anachronismen}

\section{in den platonischen Gesprächen}

von

\section{E. Zeller.}

Aus den Abhandlungen der Königl. Akademie der Wissenschaften zu Berlin 1873.

\section{Berlin.}

Buchdruckerei der Königl. Akademie der Wissenschaften (G. Vogt).

Universitäts - Strasse 8 .

1873.

In Commission bei F. Dümmler's Verlags-Buchhandlung (Harrwitz und Gossmann) 

Über

\title{
die Anachronismen
}

\section{in den platonischen Gesprächen}

von

\author{
E. Żeller.
}

Aus den Abhandlungen der Königl. Akademie der Wissenschaften zu Berlin 1873.

\section{Berlin.}

Buchdruckerei der Königl. Akademie der Wissenschaften (G. Vogt).

Universitäts - Strasse 8 .

1873.

In Commission bei F. Dümmler's Verlags-Buchhandlung

(Harrwitz und Gossmann) 
Gelesen in der Akademie der Wissenschaften am 17. Juli 1873.

Die Seitenzahl bezeichnet die laufende Pagina des Jahrgangs 1873 in den Abhandlungen der philosophisch-historischen Klasse der Königl. Akademie der Wissenschaften. 
So wenig auch die Reden des platonischen Sokrates ihrer überwiegenden Mehrzahl nach für historisch treue Berichte über die Lehre dieses Philosophen gelten können, so viele Mühe hat sich doch Platon gegeben, sie als solche erscheinen zu lassen. Er knüpft seine Gespräche an bekannte Persönlichkeiten und geschichtliche Ereignisse an; er schildert uns ausführlich bis in's einzelste die Umstände, unter denen sie gehalten und durch die sie veranlafst worden seien; er legt uns wohl auch über die Personen, von denen sie aufgezeichnet oder wiedererzählt sein sollen, und über das Verfahren derselben eine Rechenschaft ab, welche ganz den Eindruck macht, als solle sie jedem Zweifel an der Glaubwürdigkeit seiner Darstellung ausdrücklich vorbeugen.

Aber seltsam: in dem gleichen Augenblick, in welchem der Schriftsteller diese Täuschung in uns hervorruft, zerstört er sie nicht selten mit eigener Hand wieder durch jene Anachronismen, welche uns oft so grell wie möglich daran erinnern, dals wir es hier nicht mit einer geschichtlichen Überlieferung, sondern mit einer freien Dichtung zu thun haben. Für die Untersuchung über die platonischen Schriften sind diese Anachronismen von bedeutendem Werth, weil sie uns die Zeitpunkte bezeichnen, über welche die Abfassung der Gespräche, in denen sie vorkommen, nicht hinaufgerückt werden kann; aber auf Platon's schriftstellerisches Verfahren werfen sie ein eigenthümliches Licht, und die Frage 
legen sie nahe, ob und wie weit man sich in solchen Fällen, in denen wir seine Angaben an anderweitigen zuverlässigen Zeugnissen zu prüfen aufser Stand sind, auf dieselben verlassen und auch nur die Absicht, durchaus glaubwürdige und widerspruchslose Berichte zu geben, bei ihm voraussetzen darf.

Wollen wir uns nun die hieher gehörigen Stellen der platonischen Schriften im einzelnen vergegenwärtigen, so werden wir uns bei solchen Dialogen nicht aufzuhalten brauchen, deren Urheberschaft Platon mit triftigen Gründen abgesprochen wird. Wir werden uns die Frage ersparen dürfen, wie der Menexenos Sokrates und seiner angeblichen Lehrmeisterin Aspasia eine Rede in den Mund legen könne, welche die Geschichte Athen's bis zum antalkidischen Frieden, zwölf Jahre über den Tod des Sokrates und vielleicht noch weiter über den der Aspasia hinaus, verfolgt; oder wie der erste Alkibiades den Philosophen in derselben Zeit, in welcher ihn andere platonische Gespräche in einem sehr nahen, schon lange bestehenden Verhältnils zu Alkibiades zeigen, nach jahrelangem Schweigen das erste Wort an diesen seinen jungen Freund richten lassen könne. Diese Verstölse gegen die Geschichte weichen wirklich von allem, was sich bei Platon ähnliches findet, immer noch so weit ab, dafs sie unsere Überzeugung von der Unächtheit der ebengenannten Gespräche nur verstärken können.

Aber auch die ächten Werke des Philosophen zeigen in dieser Beziehung manche für uns sehr auffallende Erscheinungen. In einer bekannten Stelle des Gastmahls (193, A) erwähnt Aristophanes des arkadischen diciririos, jener Mafsregel, welche die Lakedämonier (nach Xenophon Hellen. V, 2) Ol. 98, 4. 385 v. Chr. gegen die Bewohner Mantinea's ergriffen, indem sie dieselben zwangen, ihren städtischen Verband aufzulösen, und sich in vier unbefestigte Flecken zu vertheilen. Es liegt auf der Hand, dafs sich Platon-dieses Anachronismus als eines solchen bewufst sein mufste, und auch von den ersten Lesern seiner Schrift konnte er kaum einen für unwissend genug halten, um nicht zu bemerken, dafs unmöglich in Gegenwart des Sokrates und bei der hier geschilderten Veranlassung von einem Ereignifs gesprochen worden sein könne, welches sich eben erst, 14 Jahre nach Sokrates Tod, und 32 Jahre nach der angeblichen Zeit des Mahles in Agathon's Hause, zugetragen hatte. Diese 
Hintansetzung aller chronologischen Möglichkeit und geschichtlichen Denkbarkeit findet sich nun freilich in einer Rede, welche dem Aristophanes in den Mund gelegt und von Anfang bis zu Ende mit dem Geist der aristophanischen Komödie so gesättigt, von einem so sprudelnden Humor erfüllt ist, dafs man selbst von einem so übermüthigen Spiel des Verfassers mit dem Leser in diesem Zusammenhang kaum überrascht wird, und mit allem anderen undenkbaren, das der unübertreffliche Lustspieldichter uns erzählt, am Ende auch dieses unbefangen hinnimmt. Aber daran erinnert sie uns doch immer sehr lebhaft, dafs wir hier das freieste Erzeugnifs des platonischen Geistes vor uns haben; und wenn Platon kein Bedenken trägt, uns diese Wahrnehmung so greifbar nahe zu legen, so beweist diefs, wie wenig er selbst die Reden, welche er uns berichtet, für etwas anderes, als seine eigene Composition, gehalten wissen will.

Ein zweites Beispiel des gleichen Verfahrens bietet uns der Menon. Dieses Gespräch könnte, wenn es wirklich stattgehabt hätte, nicht über Ol. 94, 3. 402 v. Chr. herabgerückt werden, da derjenige Theilnehmer an demselben, nach dem es benannt ist, schon im folgenden Jahr den Feldzug des jüngeren Kyros mitmachte, und nach dem Tod dieses Fürsten mit den übrigen Führern der griechischen Söldnerschaar umkam. Nichtsdestoweniger bezieht sich Sokrates darin auf einen Vorfall, der sich erst sieben Jahre nach diesem Zeitpunkt und vier Jahre nach seinem eigenen Tod zugetragen hat. Denn wenn hier S. 90 A von dem Thebaner Ismenias gesprochen wird, welcher unlängst die Schätze des Polykrates erhalten habe, so ist es kaum möglich, bei diesen Worten an etwas anderes zu denken, als an die bekannten Vorgänge aus dem Jahr 395 v. Chr. (Ol. 96, 2), wo der Rhodier Timokrates als persischer Agent Theben, Korinth, Argos und Athen bereiste, und durch reichliche Geldspenden, welche in Theben namentlich auch dem Ismenias zuflossen, das Zustandekommen eines Bündnisses gegen Sparta mit bestem Erfolge betrieb (Xen. Hell. III, 5, 1). Der Betrag dessen, was Ismenias damals erhielt, war ohne Zweifel vom Gerücht stark übertrieben worden; da indessen Timokrates für den angegebenen Zweck fünfzig Silbertalente mitgebracht, und nach Xenophon, wie es scheint, nicht mehr als acht bis zehn einflulsreiche Personen für sich zu gewinnen nöthig gehabt hatte, so kann an einzelne von diesen immerhin eine nach den damaligen VerAbh. der philos.-histor. Kl. 1873. Nr. 4. 
hältnissen sehr bedeutende Summe gekommen sein, und Platon kann hinreichenden Anlals gehabt haben, den Reichthum des thebanischen Partheiführers, welcher auch in der Republik I, 336, A berührt wird, in der angegebenen Weise mit dem persischen Geschenk in Verbindung zu bringen. Hat er diefs aber hier gethan, so hat er sich einen Verstofs gegen die Zeitrechnung erlaubt, der wieder unmöglich für unbewufst gehalten werden kann, und der beim ersten Erscheinen seiner Schrift, wèlches von dem Jahr 395 schwerlich weit abliegt, auch andern nicht wohl verborgen bleiben konnte.

Mit derselben Freiheit werden die Zeitverhältnisse im Gorgias behandelt. Wenn wir fragen, wann wir uns dieses Gespräch nach der Absicht seines Verfassers gehalten denken sollen, so lälst sich kaum ein anderer Zeitpunkt angeben, als der, in welchem der berühmte sicilische Redner als Gesandter zum erstenmal nach Athen kam, Ol. 88, 2. 427 v. Chr., oder höchstens eines der nächstfolgenden Jahre. Denn wenn auch aus dem Menon 71, C nicht mit Sicherheit geschlossen werden kann, dals Platon nur von Einem Besuch des Gorgias in Athen wufste, so macht doch schon der Anfang des Dialogs entschieden den Eindruck, als ob dieser Mann zum erstenmal hier auftrete, und andere Stellen verbieten uns mit Bestimmtheit, uns von dem genannten Jahr weit zu entfernen. Nikias, der 415 nach Sicilien gieng und 413 dort umkam, wird 472, A unbestreitbar noch unter den Lebenden aufgeführt, während Perikles

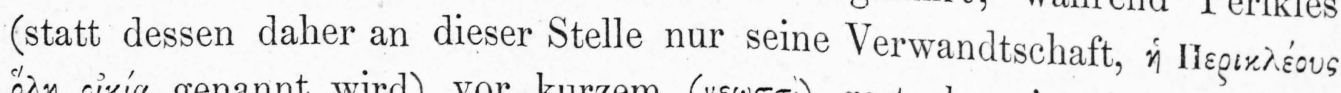
¿ $\lambda \eta$ cixice genannt wird) vor kurzem ( $v \varepsilon \omega \sigma \tau i)$ gestorben ist $(503, \mathrm{C})$; mit Alkibiades, der um 450 v. Chr. oder noch etwas früher geboren, Athen 408 für immer verliels, steht Sokrates (481, D. 482, A) noch in dem erotischen Verhältnils, welches nur einem jungen Mann gegenüber möglich war, und der Wechsel der Volksgunst, den er seit dem Beginn des sicilischen Feldzugs wiederholt erfuhr, wird hier nur als etwas, das künftig einmal kommen könne, in Aussicht genommen ${ }^{1}$ ). Neben Alkibiades wird endlich Demos, der Sohn des Pyrilampes, als ein schöner Jüngling, der Liebling des Kallikles, genannt (481, Df. 513, B); von diesem Demos

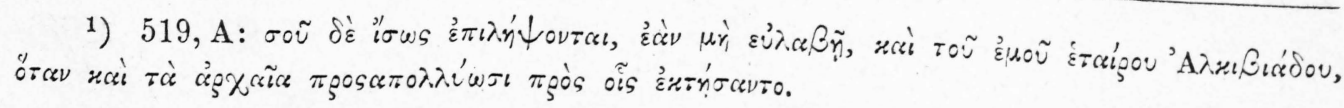


wissen wir aber aus Aristophanes' Wespen (V. 98), dafs er zur Zeit dieses Stücks, Ol. 89, 2. 422 v. Chr., eine gefeierte männliche Schönheit war. Nach allen diesen, durch das ganze Gespräch sich hindurchziehenden und mit einander durchaus übereinstimmenden Zügen läfst sich nicht bezweifeln, dafs Platon die Unterredung des Gorgias mit Sokrates in die Zeit seiner ersten Anwesenheit in Athen, oder eines der nächsten Jahre verlegen will. Aber auch hier finden sich einige Stellen, welche sich mit dieser Zeitbestimmung so wenig vertragen, dafs manche Gelehrte um ihretwillen die angebliche Zeit des Gesprächs bis in das Jahr 405 v. Chr. herabrücken zu sollen geglaubt haben. Denn es wird nicht allein der Gewaltthaten, durch welche sich der macedonische Archelaos 414 v. Chr. den Weg zum Thron bahnte, wiederholt und ausführlich gedacht (470, Dff. 479, A. Df. 525, D), sondern Sokrates erinnert auch 473, E (wiewohl noch 472, A der mit den übrigen Arginusensiegern hiugerichtete Aristokrates gegenwärtig ist) so unzweideutig wie möglich an die bekannte Episode in dem Procels der arginusischen Sieger vom Jahr 406 v. Chr., da er als Prytane der Volksleidenschaft gegenüber das Gesetz unerschütterlich festhielt; nur dals er in seiner Weise den für ihn selbst $\mathrm{SO}_{4}$ rühmlichen Vorgang in das durchsichtige Gewand einer leichten Ironie hüllt. Durch diese Wendung hat es sich Platon allerdings erspart, den auffallenden Anachronismus, den er sich hier erlaubt, mit dürren Worten auszusprechen; aber der Sache nach ist er in diesem Falle gerade so gut, wie im Gastmahl und im Menon, vorhanden.

Nicht anders verhält es sich mit dem Protagoras. Die Kritiker sind darüber ziemlich einig, dals die Zeit dieser Unterredung nach Platon's Absicht nicht über den Anfang des peloponnesischen Krieges herabgerückt werden darf. Perikles und seine Söhne, welche 429 v. Chr. von der Pest weggerafft wurden, sind hier $(315$, A. 319, E) noch am Leben; Xanthippos, der ältere der beiden Periklessöhne, der als junger Ehemann starb (Plut. Pericl. 36), ist der väterlichen Erziehung noch nicht entwachsen; von Phidias, dessen Tod in das Jahr vor dem Ausbruch des Krieges, 432 v. Chr. fällt, heifst es $(311, \mathrm{C})$, man könne bei ihm die Bildhauerkunst lernen; Sokrates ist noch ein jüngerer Mann, von dem Protagoras erst erwartet, dals er sich einen Namen machen werde (361, E); Alkibiades steht an der Schwelle des reiferen Jünglingsalters, in der Zeit 
des ersten Bartwuchses (309, A); Hippias aus Elis hält sich ruhig vor aller Augen in Athen auf, wo er sich als Peloponnesier während des Kriegs nicht blicken lassen durfte. Alle diese Züge vereinigen sich zu dem Ergebnifs, dals das Gespräch spätestens in das Jahr 432 v. Chr. verlegt sein wolle; dürfen wir mit seinen eigenen Aussagen auch noch die des Gastmahls verbinden, nach dem Sokrates in diesem Jahr mit Alkibiades vor Potidäa lag, so müssen wir noch etwas weiter, etwa bis 433 v. Chr., hinaufgehen. Aber so einleuchtend alles diefs ist, so findet sich doch daneben anderes, was hiezu nicht stimmen will. Die Unterredung spielt bekanntlich im Hause des Kallias, dieses reichen und angesehenen Gönners der Sophisten, welcher sein grolses, von seinem Vater Hipponikos ererbtes Vermögen schliefslich über dieser und andern kostspieligen Liebhabereien vergeudete. Nun sehen wir aber aus Thukydides (III, 91), dals Hipponikos noch im sechsten Jahr des peloponnesischen Kriegs, 426 v. Chr., das athenische Heer in dem siegreichen Gefecht bei Tanagra befehligte, und aus Andokides (adv. Alcib. 13 vgl. Athen. V, 218, B), dafs er zwei Jahre später, 424 v. Chr., bei Delion umkam. Soll nun doch Kallias, und nicht Hipponikos, die Sophisten, mit denen Sokrates sich besprach, bei sich aufgenommen haben, so müfste man entweder mit Schleiermacher (Pl. WW. I, a, 220) annehmen, Kallias habe während einer längeren Abwesenheit seines Vaters dessen Stelle vertreten, oder mit Munk (Ordn. d. plat. Schr. 84), er habe ein eigenes ihm von seinem Vater abgetretenes Haus bewohnt. Aber Platon selbst hat uns, wie absichtlich, beide Auswege verlegt. Dem ersten steht nicht allein der Umstand entgegen, dals das Haus, welches Schauplatz des Gesprächs ist, S. 311, A ausdrücklich als das des Kallias bezeichnet, seines Vaters dagegen als des eigentlichen Hausherrn nirgends mit einem Worte gedacht wird; sondern er wird auch durch die Bemerkung S. 315, D ausgeschlossen: Kallias habe wegen der grofsen Zahl seiner Gäste ein Gemach, welches Hippo-

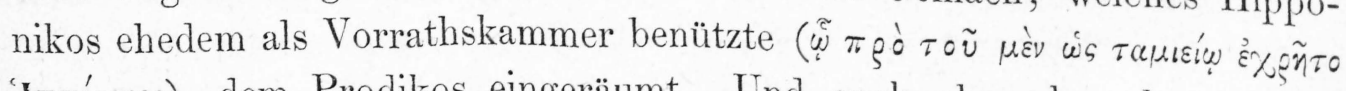
'I $\pi \pi_{0}^{\prime}$ (rkos), dem Prodikos eingeräumt. Und auch abgesehen davon kann man es sich kaum als Platon's Meinung denken, dafs Kallias während einer vorübergehenden Abwesenheit seines Vaters in dem väterlichen Hause die hergebrachte Ordnung so vollständig umgestürzt und sich in einer Weise, die selbst der Dienerschaft zu viel war $(314, \mathrm{Cf}$.), mit seiner 
Sophistenwirthschaft darin eingerichtet habe; wäre sie es aber gewesen, so hätte er es schwerlich unterlassen, mit einem Wort darauf hinzüweisen, zu welcher Rücksichtslosigkeit gegen seinen Vater ihn die Schwärmerei für die neuen Weisheitslehrer verleitet habe. Munk's Annahme würde sich zwar mit der Chronologie, so weit wir diese kennen, nothdürftig vertragen; denn da Kallias' jüngerer Halbbruder Xanthippos, wie bereits bemerkt wurde (S. 83), einige Zeit vor seinem Tode, der 429 v. Chr. erfolgte, bereits verheirathet war (Plut. Pericl. 24. 36), so kann es am Ende Kallias selbst drei bis vier Jahre früher auch schon gewesen sein. Dagegen scheitert dieselbe daran, dafs das Haus des Kallias S. 337, D von dem Sophisten Hippias das grölste und reichste Haus der Stadt genannt wird; denn so lange Hipponikos am Leben war, konnte diefs wohl von seinem eigenen Haus, aber nicht von dem seines Sohnes gesagt werden. Der Anachronismus lälst sich daher in keiner Weise beseitigen. Für unbewufst und unwillkührlich wird er aber so wenig wie die meisten früher angeführten gehalten werden können; denn Platon, selbst einem der ältesten attischen Adelsgeschlechter angehörig, kann mit der neuesten Geschichte seiner Vaterstadt und der ersten Häuser in derselben nicht unbekannt g̀nug gewesen sein, um nicht zu wissen, dal's Hipponikos, der Sieger von Tanagra, nächst Perikles einer von den reichsten und angesehensten Männern Athen's, die ersten Jahre des peloponnesischen Kriegs überlebt hatte. Den Grund dieses Anachronismus werden wir wohl in künstlerischen Rücksichten zu suchen haben. Denn einerseits gab es kein Haus in Athen, welches sich besser, als das des Kallias, zum Schauplatz für eine so vollständige Versammlung der angesehensten Sophisten geeignet hätte; andererseits lälst sich nicht verkennen, dals die ganze Haltung des Gesprächs und die Schilderung des Verhältnisses zwischen Sokrates und Protagoras eine andere geworden wäre, wenn Sokrates dem Sophisten als reiferer, durch seine eigene Lehrthätigkeit schon zu einer anerkannten Stellung gekommener Mann gegenüber träte, und dafs sich ein Alkibiades und Kritias gleichfalls nach dem Frieden des Nikias nicht mehr ebensogut zu Repräsentanten der lernbegierigen Jugend Athen's eigneten, wie zehn Jahre früher. Ein neuerer Schriftsteller hätte nun vielleicht trotzdem, um sich mit der Zeitrechnung nicht in Widerspruch zu setzen, die Unterredung im Hause des Kallias in jene spätere Periode verlegt, und 
auch Platon hätte diefs thun können, wenn er gewollt hätte; aber doch ist er viel zu sehr Dichter, als dafs ihn dieses Bedenken abhalten konnte, so zu verfahren, wie er verfahren ist, wenn er einmal den Plan gefafst hatte, uns die Sophistik auf dem Höhepunkt, den sie unmittelbar vor dem Ausbruch des peloponnesischen Kriegs, in der Blüthezeit des Protagoras einnahm, und ihr gegenüber den ächten Philosophen noch im Werden, im Suchen der wahren Tugendlehre begriffen, darzustellen. Um so weniger haben wir ein Recht, an einem zweiten, weit unerheblicheren Anachronismus, der beiläufigen Beziehung auf die "Wilden" des Pherekrates $(327, \mathrm{D}$ ), die Ol. 89, 4. 420 v. Chr. aufgeführt wurden (Athen. V, $218, d)$, Anstols zu nehmen und mit Schleiermacher (I, a, 220) noch eine weitere, frühere Aufführung dieses Stücks zu vermuthen. Ebensowenig hätte es auf sich, wenn der Tragiker Agathon hier etwas zu alt gemacht sein sollte. Die Art, wie er nach seinem ersten dramatischen Siege, 417 v. Chr., im Gastmahl $(175, \mathrm{E} .223, \mathrm{~A})$ geschildert wird, macht es allerdings zweifelhaft, ob er 15-16 Jahre früher das Knabenalter schon überschritten hatte, was man doch nach Prot. 315, D annehmen mufs. Dals aber Platon die Unterredung zwischen Sokrates und Protagoras erst in oder nach $424 \mathrm{v}$. Chr. setzen wolle, kann man daraus um so weniger schliefsen, da diese Voraussetzung zu der Darstellung des Gastmahls gleichfalls nicht pafst.

Nach allem bisherigen dürfte es uns nun nicht überraschen, auch in der Republik auf chronologische Schwierigkeiten zu stofsen, welche sich nicht vollständig beseitigen liefsen. Indessen fragt es sich erst, ob wir hier wirklich erhebliche Anachronismen vor uns haben. Die Ansichten der Gelehrten gehen bekanntlich in Beziehung auf die Zeit, welche Platon diesem Gespräch anweist, aufserordentlich weit auseinander; und wollen wir auch von der Meinung absehen, dafs dasselbe von seinem Verfasser in das vierte Jahr der 83. Olympiade, 445 v. Chr., oder ein noch früheres, d. h. in einen Zeitpunkt verlegt werde, in welchem Sokrates unmöglich schon zum Führer einer so grofsartig angelegten, alle Höhen und Tiefen des platonischen Systems umfassenden Unterredung gemacht werden konnte, welche sich aber auch sonst mit der Chronologie der in dem Gespräch auftretenden und erwähnten Personen schlechterdings nicht verträgt, so besteht doch immer noch unter den gründ- 
lichsten Kennern der platonischen Schriften ein auffallender Zwiespalt. Denn während Hermann ${ }^{1}$ ) unter Steinhart's ${ }^{2}$ ) Beistimmung die angebliche Zeit des Dialogs in d. J. 429 v. Chr., Ol. 87, 3, setzt, geht Böckh ${ }^{3}$ ) mit derselben bis zu Ol. 91, 3. 412 v. Chr. und Susemihl ${ }^{4}$ ) noch zwei Jahre weiter herab. Der Hauptgrund dieser Differenz liegt aber freilich nicht darin, dals die platonischen Angaben unter einander, sondern darin, dafs sie mit den Angaben späterer Schriftsteller, - des Cicero, des Dionys von Halikarnals, des angeblichen Plutarch in der Schrift über die zehen Redner, des Photios und Suidas sich nicht in Einklang bringen lassen wollen. Dürften wir diesen Schriftstellern Glauben schenken, so mülste Kephalos, der Vater des Lysias, welcher uns nebst seinen Söhnen im Eingang des platonischen Staates begegnet, schon vor der Gründung von Thurii, d. h. vor Ol. 84, 1 (444 v. Chr.), gestorben sein, nachdem er dreifsig Jahre in Athen gewohnt hatte. Allein diese Annahme läfst sich nicht allein mit der platonischen Darstellung, sondern auch mit der eigenen Aussage des Lysias (adv. Eratosth. 4) nicht vereinigen; die späteren Überlieferungen über das Leben des Lysias sind mithin offenbar von ungeschichtlichen Bestandtheilen nicht frei. Dann hat man aber kein Recht mehr, denselben Zeugen, deren Glaubwürdigkeit man bei einem Theil ihrer Angaben preiszugeben nicht umhin kann, bei einem anderen Theil derselben ein solches Vertrauen zu schenken, dafs man ihnen zu gefallen Platon etwas anderes sagen läfst, als er offenbar sagt. Hält man sich an seine Darstellung als solche, so lälst sich nicht bezweifeln, dafs die Unterredungen, welche die Republik berichtet, seiner Absicht nach nicht früher fallen können, als das Ende des sicilischen Feldzugs; und selbst über die von Böckh und Susemihl hiefür angenommenen Jahre dürfte noch etwas herabzugehen, und ihre Zeit etwa in Ol. 92, 4 (409 v. Chr.) oder auch in das nächstfolgende Jahr zu setzen sein. Unter den Personen des Gesprächs spielen Adeimantos und Glaukon, die Söhne Ariston's, eine hervorragende Rolle. Beide sind noch

1) Vindiciae Plat. II (Disputatio de reip. Plat. temp. 1839). Gesch. u. Syst. d. Plat. I, 695, 683.

2) Platon's Werke V, 58 f. $672 \mathrm{f}$.

3) Index lect. Berol. hib. 1838 aest 1839. 1840.

4) Genet. Entw. d. plat. Phil. II, 76 ff. 


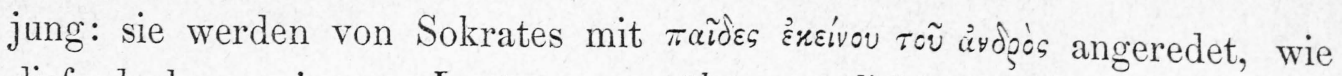
diefs doch nur jungen Leuten gegenüber möglich war; Glaukon's Liebhaber hat auf sie - offenbar erst vor kurzem, und nicht etwa schon vor Jahrzehenden - ein Gedicht gemacht $(368, \mathrm{~A})$; und Adeimantos rechnet sich selbst im Vergleich mit Sokrates zu einer jüngeren Generation, wenn er II, 367, A der Forderung, dals der Vorzug der Gerechtigkeit vor der Ungerechtigkeit an dem inneren Wesen dieser entgegengesetzten sittlichen Zustände, nicht blos an ihren Folgen, nachgewiesen werden sollte, die Worte beifügt: "Hättet ihr alle von Anfang an in diesem Sinn mit uns geredet, und uns von früh auf überzeugt, so brauchten wir einander nicht durch gegenseitige Überwachung vom Unrecht abzuhalten, sondern jeder wäre sein eigener Wächter". Diels palst nun vollkommen, wenn mit diesem Brüderpaar niemand anders gemeint ist, als die bekannten Brüder Platon's, auf die ja auch schon der Name ihres Vaters mit solcher Bestimmtheit hinweist, dals wir unbedingt erwarten müfsten, wenn Platon bei unserem Glaukon und Adeimantos an andere, als seine Brüder, und bei ihrem Vater Ariston an einen andern als seinen Vater gedacht hätte, so würde er diefs irgendwie angedeutet haben, da ja doch auch schon seinen Zeitgenossen, wenn sie von einem Glaukon und Adeimantos, den Söhnen Ariston's, hörten, die Brüder und der Vater Platon's ungleich näher liegen mulsten, als irgend welche andern, einer früheren Zeit angehörige Personen. Auch jene üvdoseic, welche II, 357, A an Glaukon gerühmt wird, sammt dem vorgreifenden und etwas vorlauten Wesen, das sowohl hier als I, 328, B bei ihm hervortritt, stimmt vollkommen zu dem, wae Xenophon Mem. III, 6 über Glaukon berichtet, sobald wir uns nur erinnern, dals es in der Republik eben sein Bruder ist, der über ihn spricht; und mit der Schlacht bei Megara, in der die Söhne Ariston's sich auszeichneten, kann recht wohl das von Diodor XIII, 65 erwähnte Treffen des Jahrs 409 v. Chr. Ol. 92, 4 gemeint sein, in dem ein kleines athenisches Heer über eine ihm bedeutend überlegene feindliche Macht einen glänzenden Sieg erfocht; ein Treffen, an dem nach der damaligen Lage der Stadt auch so junge Leute, wie diefs wenigstens Glaukon, Platon's jüngerer Bruder, damals jedenfalls noch war, theilgenommen haben können. Setzt man dagegen mit Hermann die angebliche Zeit des Gesprächs zwanzig Jahre früher, so mufs man unter dem Glaukon 
und Adeimantos desselben zwei ältere Verwandte Platon's verstehen, deren Dasein man auch aus dem Eingang des Parmenides erschliefsen zu können glaubte. Aber von diesen fehlt nicht allein jede anderweitige Spur, sondern auch der Parmenides giebt uns, wie Böckh ausreichend gezeigt hat, zu ihrer Annahme kein Recht, ja er kann sich gar nicht auf Männer aus einer so frühen Generation beziehen, da die Zeit, in welcher der Klazomenier Kephalos (mit dem Vater des Lysias, dem Syrakusaner, nicht zu verwechseln) in Athen mit Adeimantos und Glaukon zusammentrifft, später gedacht sein mufs, als die Herrschaft der Dreifsig und der Tod des Sokrates. Wollen wir aber auch davon absehen, und wollen wir uns die weitere Unwahrscheinlichkeit gleichfalls gefallen lassen, dals nicht blos diese beiden mütterlichen Verwandten Platon's, sondern auch ihr Vater, seinen beiden Brüdern und seinem Vater gleichnamig gewesen wären, so stellen sich dieser Annahme immer noch die grölsten Schwierigkeiten in den Weg. Denn jener ältere Glaukon und Adeimantos mülsten, wenn man bei dem Treffen, in dem sie sich auszeichneten, mit Hermann und Steinhart an die bekannte Schlacht des Jahrs 456 v. Chr. (Thuc. I, 105) denken wollte, um mehrere Jahre älter gewesen sein, als Sokrates, was sich mit der Darstellung unserer Schrift schlechterdings nicht verträgt; und es mülste bei der Erwähnung dieses Treffens doch irgendwie darauf hingedeutet sein, dafs es sich hier um etwas handle, was sich vor mehr als einem Vierteljahrhundert zugetragen hatte. Später aber zeigt sich bis über das Jahr herab, in welches Hermann das Gespräch verlegt, kein Vorfall mehr, der ihnen zu jener Auszeichnung Gelegenheit geben konnte. Mit Recht schliefst ferner Böckh

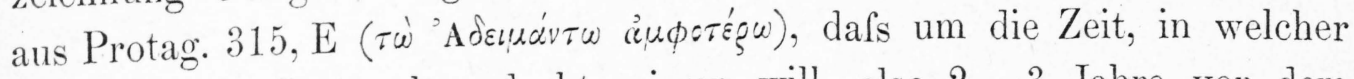
Platon dieses Gespräch gedacht wissen will, also $2-3$ Jahre vor dem von Hermann für die Republik angenommenen Zeitpunkt, aus dem Kreise der mit Sophisten verkehrenden vornehmen Jugend in Athen dem Platon aufser den beiden dort genannten Adeimantos kein dritter bekannt war. Wie man sich endlich die Theilnahme des voraussetzlichen älteren Adeimantos und Glaukon an einer Schlacht bei Megara erklären möchte: jedenfalls wäre Sokrates Ol. 87, 3 ein Mann von kaum vierzig Jahren gewesen, der jenem Brüderpaar, und namentlich dem Adeimantos, nicht Abh. der philos.-histor. Kl. 1873. Nr. 4. 
wohl in der Weise, wie es hier geschieht, als ein Angehöriger der älteren Generation gegenübergestellt werden konnte, und der auch gegen Kephalos sich schwerlich so äufsern würde, wie er diefs S. 328, D thut. Weiter begegnet uns im Eingang der Republik der eben genannte Kephalos, der Vater des Lysias, als ein hochbetagter, der äufsersten Grenze

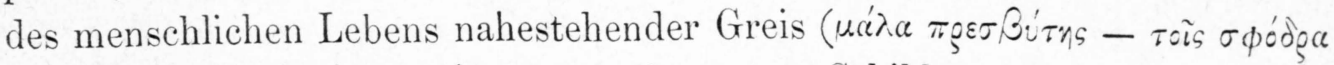

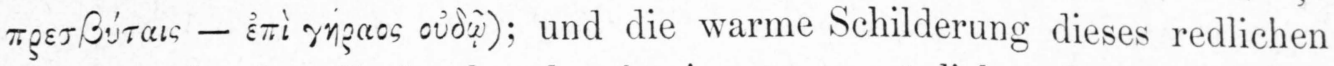
Alten macht den Eindruck, als ob sie aus persönlicher Erinnerung geflossen sei. Doch kann dieses Merkmal täuschen; entscheidender ist ein anderes. Kephalos erwähnt $(329, \mathrm{~B})$, er sei einmal zugegen gewesen,

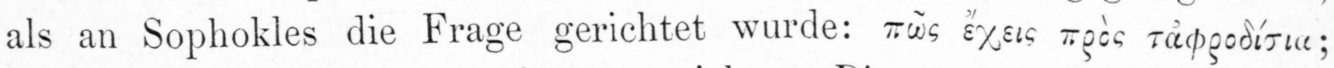
worauf dieser die bekannte Antwort giebt. "Dieses nun schien mir schon damals wohlgesprochen zu sein, und jetzt noch mehr ${ }^{1}$ ). Denn allerdings

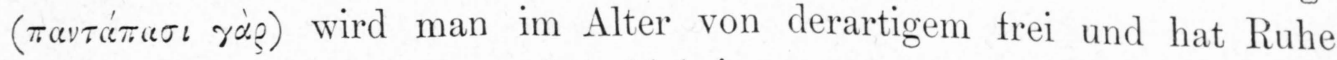
davor". Es liegt am Tage, dals hiebei vorausgesetzt wird, Kephalos sei jünger gewesen, als Sophokles; denn nur defshalb kann er den Eindruck, welchen der Ausspruch des letzteren beim ersten Hören auf ihn gemacht hat, in der Folge bestätigt finden, weil er über das, was er damals noch nicht aus eigener Erfahrung kannte, die Beruhigung der Leidenschaften im höheren Alter, inzwischen Erfahrungen gesammelt hat. Denken wir

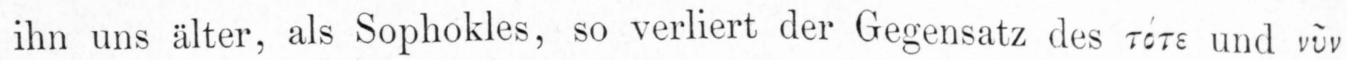
seine Bedeutung, und diese ganze Bemerkung wird unverständlich. Nun war aber Sophokles in der Zeit, in die Hermann unser Gespräch setzt, höchstens 67 Jahre alt; Kephalos mülste demnach etwas jünger, etwa ein angehender Sechziger, gewesen sein. Wäre er aber auch gleich alt gewesen, so kann ein 67 jähriger Mann noch immer nicht als ein solcher dargestellt werden, welcher der letzten Grenze des Greisenalters nahe steht. - Unter den Jüngeren, welche der Unterredung anwohnen, treffen wir aufser andern Nikeratos, den Sohn des Nikias. Eben dieser wird aber im Laches $(180$, C. 200, D), einige Zeit nach der Schlacht bei Delion, als ein junger Mensch genannt, für den noch Lehrer in der Musik

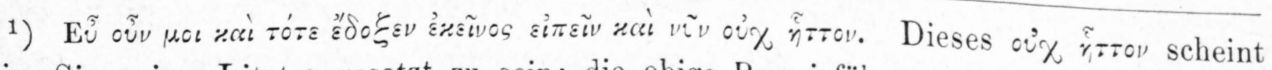
mir im Sinn einer Litotes gesetzt zu sein; die obige Beweisführung gilt aber auch, wenn man es einfach mit, ebenso" übersetzt. 
und anderen Fächern gesucht werden. Er könnte daher um 430 v. Chr. kaum mehr als zwölf Jahre alt gewesen sein und unmöglich so, wie hier $(327, \mathrm{C})$, selbständig an der Gesellschaft der Männer theilnehmen. Wenn ferner mit Charmantides dem Päanieer $(328, \mathrm{~B})$ derselbe gemeint ist, welcher später zu den älteren Schülern des Isokrates gehörte (Isocr. $\pi$. ảvtidós. 93), so würde sich auch dieses nur mit unserer (beziehungsweise Böckh's) Annahme über die Zeit des Gesprächs vertragen. Durch dieselbe wird auch der grobe Anachronismus, der andernfalls in der Erwähnung des Thebaners Ismenias als eines reichen und einflufsreichen Mannes $(336, A)$ liegen würde, gehoben oder doch auf ein kleinstes zurückgeführt; denn dieser Ismenias, welcher 383 v. Chr. als Polemarch hingerichtet wurde (Xen. Hellen. V, 2, 25 ff.), kann unmöglich sechs- oder siebenundvierzig Jahre früher ein einflulsreicher Mann gewesen sein, wogegen diefs um 410 v. Chr. allerdings möglich erscheint, wenn er auch erst später genannt wird. Wenn endlich unser Gespräch von Platon selbst auf den Abend des Tages verlegt wird, an welchem die Bendideen unter Betheiligung thracischer Festgäste, die einen eigenen Aufzug veranstalten, zum erstenmal in Athen gefeiert wurden, so nützt uns dieses Datum zwar nicht viel, da nichts darüber bekannt ist, wann jenes Fest eingeführt wurde; aber Herman's Vermuthung, dafs diefs nach der ersten Verbindung Athen's mit Sitalkes ${ }^{1}$ ) geschehen sei, kann man mit gleichem Recht eine beliebige andere entgegenstellen, und wenn er es unwahrscheinlich findet, dafs unmittelbar nach der sicilischen Niederlage ein solches Fest in Athen gefeiert werden konnte, so ist es nicht eben wahrscheinlicher, dafs diefs Ol. 87,3 geschehen sein sollte, während in Athen und namentlich im Piräeus die Pest wüthete (Thuc. II, 47 f.); derselbe Umstand verbietet aber auch, hiefür ein Jahr weiter, in das zweite Jahr der genannten Olympiade, hinaufzugehen, denn auch in diesem Jahr war nach Thuc. a. a. O. im Thargelion, der Zeit des Bendisfestes, die Seuche bereits ausgebrochen; während andererseits die Verbinduk̂g mit Sitalkes im vorangehenden Jahre, das sich ohnedem als das Anfangsjahr des Kriegs gegen Sparta zu neuen Festen gleichfalls nicht eignete, noch nicht

1) Thuc. II, 29, wo aber von dem, was Hermann (Reip. Plat. temp. 12) in dieser Stelle findet, dafs thracische Söldner nach Athen geschickt worden seien, nichts steht. 
angeknüpft war. Von diesem Punkt aus lälst sich daher keine Aufklärung der Frage erwarten, und man kann ebensogut annehmen, das thracische Fest habe sich erst weit später in Athen eingebürgert. Es zeigt sich so, dafs alle Andeutungen, die Platon selbst über die Zeit giebt, in welcher wir uns die Unterredungen der Republik gehalten denken sollen, sich mit unserer Annahme vertragen, ohne uns in irgend eine ernstliche chronologische Schwierigkeit zu verwickeln, sobald wir nur darauf verzichten, sie mit den offenbar unzuverlälsigen Angaben späterer Schriftsteller über das Leben des Lysias in Einklang zu bringen. Dafs aber Platon seinen Bruder Glaukon dem Sokrates gegenüber Gedanken aussprechen lälst, welche wir diesem, von Xenophon (Mlem. III, 6) als sehr unreif geschilderten jungen Manne wenigstens in jener Zeit nicht zutrauen können, ist nur dieselbe Freiheit, welche er sich in allen seinen Gesprächen erlaubt, unter die Anachronismen kann man diefs strenggenommen nicht rechnen.

Nicht so einfach liegt die Frage nach der Chronologie des Parmenides. Denn in dem zwar, was uns Platon in dem ersten, zur Einrahmung der Hauptverhandlung dienenden Gespräch über den Klazomenier Kephalos, über Adeimantos und Glaukon und ihren Halbbruder Antiphon mittheilt, hatte er keinen Grund, sich mit den thatsächlichen Zeitverhältnissen in Widerspruch zu setzen, und wenn es dennoch geschehen wäre, hätten wir kein Mittel, es nachzuweisen. Anders verhält es sich dagegen mit dem Besuch des Parmenides und Zenon in Athen und mit der Berührung, in welche Sokrates damals nicht blos nach unserem Gespräch, sondern auch nach dem Theätet $(183, \mathrm{E})$ und Sophisten $(217, \mathrm{C})$, mit diesen beiden Philosophen gekommen sein soll. Da die Reden, welche nach Platon bei dieser Gelegenheit gewechselt wurden, den handelnden Personen selbstverständlich nur von ihm in den Mund gelegt werden, da Sokrates nach allem, was wir von ihm wissen, die wissenschaftliche Einwirkung, die er unserer Darstellung zufolge von Parmenides erfahren hätte, nicht erfahren haben kann, da also der Kern der ganzen Erzählung jedenfalls erdichtet ist, so haben wir nicht die geringste Bürgschaft dafür, dals es sich mit den äufseren Umständen anders verhalte, durch welche diese von Platon erdichtete wissenschaftliche Verhandlung herbeigeführt wor- 
den sein soll. Platon wollte nachweisen, dafs die Lehre des Parmenides durch ihre eigene dialektische Consequenz in Antinomieen verwickle, die nur mittelst einer weiter geführten Spekulation, nur auf dem Boden der Ideenlehre gelöst werden können; er wollte aber zugleich auch in dieser. dialektischen Erörterung der allgemeinsten metaphysischen Begriffe das Mittel aufzeigen, um die Ideenlehre selbst genauer zu bestimmen und ihren Schwierigkeiten zu entgehen; er wollte mit Einem Wort die Fortbildung der parmenideischen Philosophie zur sokratischen Begriffsphilosophie, so wie er diese auffalst, ebendamit aber auch die Ergänzung der sokratischen Lehre durch die parmenideische, darstellen. Um nun an's Licht treten zu lassen, dafs jene Fortbildung der parmenideischen Ontologie ihre eigene, immanente Fortbildung sei, erschien es ihm am angemessensten, die dialektische Erörterung ihrer Grundbegriffe, welche er ohnediefs für Sokrates weniger geeignet fand, dem Parmenides selbst in den Mund zu legen; um andererseits durch dieselbe auf die sokratische Begriffsphilosophie als ihre Consequenz hinzuweisen, und auch hier, wie immer, an die Person seines Lehrers anzuknüpfen, lälst er sie dem jugendlichen, über seinen eigenen Standpunkt noch nicht mit sich in's reine gekommenen Sokrates von ihm vortragen; ähnlich wie er auch im Sophisten und im Politikos die eleatische Dialektik, im Timäos die pythagoreische Naturphilosophie mit der Sokratik dadurch in Verbindung bringt, dafs er von Mitgliedern der eleatischen und pythagoreischen Schule dem Sokrates mittheilen läfst, was er diesem selbst in den Mund zu legen Bedenken trägt. Soll aber Parmenides mit Sokrates in dieser Weise verkehrt haben, so mufs er nach Athen gekommen sein, denn den Sokrates als jungen Menschen nach Elea reisen zu lassen, wäre doch gar zu unwahrscheinlich und mit Platon's sonstiger Darstellung zu unvereinbar gewesen; und wenn jenes Zusammentreffen selbstverständlich nur in einen Zeitpunkt fallen kann, in welchem Sokrates noch sehr jung und Parmenides schon alt war, so darf der letztere doch noch nicht so alt gewesen sein, dafs ihm eine so anstrengende Rede- und Denkarbeit, wie wir sie hier finden, nicht mehr zugemuthet werden konnte. So erzählt uns denn Platon, Parmenides sei, schon betagt, etwa fünfundsechzigjährig, in Begleitung Zenon's in Athen mit Sokrates zusammengetroffen, welcher da- 


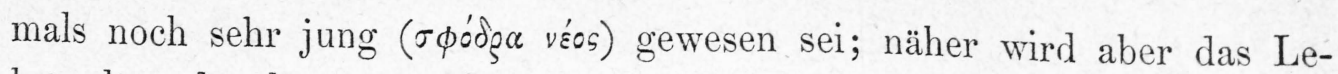
bensalter des letzteren nicht bezeichnet, wie wenn der Leser ausdrücklich von einem allzu genauen Nachrechnen abgehalten werden sollte. Läfst sich nun auch die Möglichkeit nicht bestreiten, dafs Parmenides in seinen späteren Jahren nach Athen kam, so kann man doch die Wirklichkeit dieser Thatsache nicht mit dem Zeugnifs eines Schriftstellers beweisen, der seinerseits gar nicht den Anspruch auf die Glaubwürdigkeit des Geschichtschreibers macht, und der auch dann, wenn ihm gar keine auf dieses Faktum bezügliche Überlieferung vorlag, in künstlerischen Motiven eine ausreichende Veranlassung hatte, es zu erfinden; man kann nicht behaupten, wiewohl alle die Reden, welche er zwischen Parmenides und Sokrates gewechselt werden läfst, sein eigenes Werk seien, hätte er dieselben doch nicht in dieser Weise einführen können, wenn Sokrates nicht wirklich mit Parmenides zusammengekommen, oder wenigstens dieser nach Athen gekommen wäre. Denn wer uns in der Hauptsache ganz und gar eine freie Dichtung giebt, von dem kann man weder erwarten noch verlangen, dafs er uns in den mit dieser Hauptsache auf's engste verbundenen Nebensachen einen zuverläfsigen historischen Bericht gebe. Man kann daher unsere Erzählung auch nicht benützen, um die Lebenszeit des Parmenides genauer zu bestimmen. Platon hätte ja freilich seinen Sokrates mit Parmenides nicht zusammenbringen können, wenn beide anerkanntermafsen durch einen langen Zeitraum getrennt waren. Aber wenn die Lebenszeit des Parmenides wirklich an die des Sokrates heranreichte, - wie diefs ganz abgesehen von unserer Schrift feststeht - so läfst sich nach allem, was über Platon's Behandlung der Zeitverhältnisse im bisherigen nachgewiesen ist, nicht voraussetzen, dafs er Anstand genommen hätte, den eleatischen Philosophen um zehen oder zwanzig Jahre jünger zu machen, als er war, falls er diesen Anachronismus zu seiner philosophischen Dichtung nöthig hatte. Wissen wir doch nicht im geringsten, ob seine Leser und er selbst über das Zeitalter des Parmenides genau genug unterrichtet waren, um ein persönliches Zusammentreffen desselben mit Sokrates für chronologisch unmöglich zu halten. Aber wenn er auch noch so bestimmt gewufst hätte, dafs Sokrates in dem Zeitpunkt, als Parmenides 65 Jahre alt war, noch ein Kind oder viel- 
leicht noch gar nicht geboren gewesen sei, so würde ihn diels, nach seinem sonstigen Verfahren zu urtheilen, keinen Augenblick abgehalten haben, die Sache so darzustellen, wie er sie darstellt, wenn diese Darstellung seiner künstlerischen Absicht am besten entsprach; und man kann nicht sagen, wenigstens so weit habe Platon in der Fiktion nicht gehen können, dals dieselbe zur historischen Unmöglichkeit wurde. Giebt es denn eine gröfsere historische Unmöglichkeit, als die, dafs Sokrates beim Beginn seines Jünglingsalters mit dem Haupte der eleatischen Schule die Reden wechselte, welche Platon ihnen in den Mund legt? Aber die historische, und so auch die chronologische Unmöglichkeit war für unsern Philosophen überhaupt, wie wir uns zur Genüge überzeugt haben können, kein unübersteigliches Hindernifs, wenn sich ihm eine Darstellung aus anderweitigen Gründen empfahl.

Schwieriger ist es, aus diesem Gesichtspunkt den Anachronismus zu erklären, der uns in den Gesetzen I, 642, D begegnet, wenn hier gesagt wird, Epimenides der Kretenser sei zehen Jahre vor dem Beginn der Perserkriege nach Athen gekommen, habe hier gewisse vom Orakel befohlene Opfer dargebracht, und vorausgesagt, dafs die Perser erst in zehen Jahren Athen angreifen, dann aber eine Niederlage erleiden werden. Den groben Rechnungsfehler von fast hundert Jahren, den diese Angabe enthält, könnte Platon unmöglich aus Unwissenheit begangen haben; denn das konnte doch keinem gebildeten Athener und am wenigsten einem Platon unbekannt sein, dals Epimenides in dem wichtigsten Wendepunkt der älteren Geschichte seiner Heimath, in den Jahren, welche der solonischen Gesetzgebung unmittelbar vorangiengen, durch die Sühnung der kylonischen Blutschuld Athen von schwerem Fluch nnd verderblichem Bürgerzwist befreit und bei dieser Gelegenheit jene Opfer, deren unsere Stelle erwähnt, dargebracht hatte. Andererseits sieht man nicht die entfernteste Veranlassung, die Platon haben konnte, von der geschichtlichen Überlieferung abzuweichen, nicht den geringsten Vortheil, den diese Abweichung seiner Darstellung brächte; denn wenn Kleinias der Kretenser die Verbindung seines Hauses mit Athen von der Anwesenheit des Epimenides in dieser Stadt herleitet, so konnte diefs gleich gut geschehen, ob die letztere in die Zeit vor den Perserkriegen, oder 
ob sie, mit der Überlieferung übereinstimmend, in die Zeit Solon's gesetzt wurde. Und doch läfst sich der Anachronismus auf keine Weise beseitigen. Man hat vorgeschlagen, die Zahl zu ändern; allein diefs verbietet nicht blos das zweimalige Vorkommen dieser Zahl, sondern noch bestimmter die Bemerkung: als Epimenides in Athen war, habe man sich dort bereits vor einem persischen Angriff gefürchtet, was doch vor dem jonischen Aufstand kaum möglich, und bei seiner wirklichen Anwesenheit in Athen, welche früher fällt, als die Gründung des persischen Reiches durch Kyros, absolut unmöglich war. Man könnte vielleicht bei unserem Epimenides an einen gleichnamigen jüngeren Propheten dieses Namens denken; oder man könnte den uns bekannten mit Rücksicht auf das fabelhafte Alter und den sechszicjährigen Schlaf, welche die Sage ihm beilegt, nach seinem Erwachen ein zweitesmal nach Athen kommen lassen. Aber der erste von diesen Auswegen wird uns durch die Erwägung abgeschnitten, dals nicht allein wir nur Einen Epimenides kennen, sondern auch von den griechischen Lesern unserer Schrift keiner, wenn er von dem kretensischen Weihepriester und Wahrsager Epimenides hörte, hiebei an einen andern, als den sagenberühmten Zeitgenossen Solon's denken konnte, dals daher Platon, wenn er einen anderen meinte, diels nothwendig hätte sagen müssen. Auch in dem zweiten Fall sollte man aber erwarten, dafs der Verfasser, wenn ihm die Sage von einer zweimaligen Anwesenheit des Epimenides in Athen vorlag, bei Erwähnung der zweiten an die erste, allbekannte, wenigstens mit einem Wort erinnert hätte. Allein von einem solchen wiederholten Besuch Athen's weifs keiner von den sonstigen Berichten über Epimenides; sie behaupten vielmehr ausdrücklich, er sei bald nach der Sühnung der kylonischen Blutschuld gestorben, und seinen sechzig- oder siebenundfünfzigjährigen Schlaf verlegen sie in die erste Zeit seines Lebens; erst nach demselben soll er als Wahrsager aufgetreten sein (Diog. I, 109. 111. Suid. 'Eriı.); und da er schon bei Xenophanes, um den Anfang des fünften vorchristlichen Jahrhunderts, als eine sagenhafte, der Vergangenheit angehörige Person erscheint, Diog. 111), ist es nicht wahrscheinlich, dals eine dem Verfasser der Gesetze vorliegende Überlieferung sein Leben bis in diese Zeit selbst herabreichen liefs. Wir haben also hier einen unläugbaren, 
ebenso auffallenden als unmotivirten Verstofs gegen die Zeitrechnung. Nun giebt es freilich auch für diesen Anachronismus eine Erklärung; nur nicht, so lange wir ihn Platon selbst zuschreiben. Wie hier von Epimenides erzählt wird, er habe bei Gelegenheit eines Opfers, das er in Athen darbrachte, verkündet, dals eine drohende schwere Gefahr (der persische Angriff) erst in zehen Jahren eintreten werde, so heifst es im Gastmahl $(201$, D) von Diotima, sie habe bei einem Opfer vor der Zeit der grolsen Pest für Athen einen Aufschub der Krankheit auf zehn Jahre erwirkt. Es ist eine naheliegende Vermuthung, dafs es gerade diese Stelle gewesen sei, welche der der Gesetze zum Vorbild gedient hat. Aber von Platon freilich lälst sich schwer glauben, dafs er sich selbst so ungeschickt nachgeahmt haben sollte; und so wird unsere Stelle immer eine nicht ganz unerhebliche Stütze der Annahme bilden, dafs die Gesetze, wenn auch ihrem Hauptinhalt nach ein Werk Platon's, doch ihre letzte Gestalt nicht von ihm selbst erhalten haben, dafs vielmehr die Angabe ${ }^{1}$ ), nach welcher diese Schrift erst nach Platon's Tode aus seinen noch auf Wachstafeln befindlichen, also noch nicht zur abschliefsenden Reinschrift gediehenen Aufzeichnungen von dem Opuntier Philippos herausgegeben wurde, nicht ohne Grund sei, und dafs sich dieser Herausgeber Änderungen und Erweiterungen des platonischen Textes, dessen Beschaffenheit sein Eingreifen vielleicht stellenweise sogar nothwendig machte, erlaubt habe. Dieser Thätigkeit des Herausgebers mag auch unser Anachronismus seine Entstehung verdanken ${ }^{2}$ ); Platon selbst lä (st er sich kaum zutrauen.

Beschränken wir uns auf diejenigen, welche unzweifelhaft von Platon selbst herrühren, so zeigt sich, dafs dieser Schriftsteller mit der

1) Bei Diog. III, 37. Suid. фiroropos.

2) Er liefse sich wirklich ohne Änderung in den Worten des überlieferten Textes, durch blofse Auswerfung einiger Worte und Zeilen, entfernen, wenn man a. a. O. schrei-

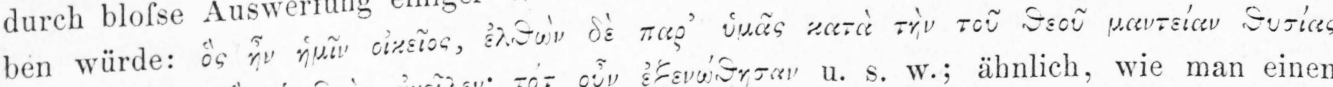

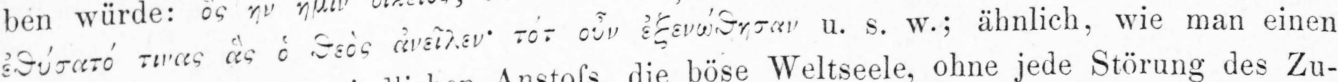
zweiten, sonst unüberwindlichen Anstols, die böse Weltseele, ohne jede Störung des Zusammenhangs entfernen könnte, wenn man die Stelle X, 896, Eff. von den Worten: Micu

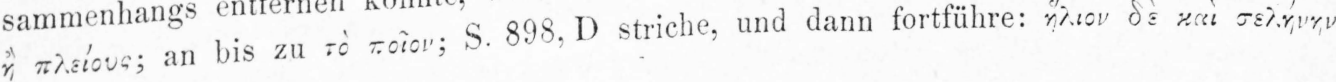
u. s. W.

Abh. der philos.-histor. Kl. 1873. Nr. 4. 
Zeitrechnung, wie mit der Geschichte überhaupt, zwar nicht selten sehr frei umgeht, dal's sich aber die Freiheiten, welche er sich in dieser Beziehung erlaubt, theils auf einzelne untergeordnete Punkte beschränken, theils da, wo sie in die Composition eines Gesprächs tiefer eingreifen, in höheren, künstlerischen Rücksichten ihre genügende Begründung finden. Diese Wahrnehmung gewährt nun für die Betrachtung der platonischen Schriften einen doppelten Vortheil. Einerseits giebt sie uns einen Masstab zur Beurtheilung solcher Anachronismen an die Hand, von denen es fraglich ist, ob wir sie Platon selbst zuschreiben dürfen, was für die Kritik einzelner unter seinem Namen überlieferter Schriften nicht ohne Werth ist. Andererseits warnt sie uns für die Fälle, in welchen Platon unsere einzige Quelle für eine geschichtliche Nachricht, z. B. aus dem Leben des Sokrates, ist, ebenso vor unkritischer Leichtgläubigkeit, we vor einer zu weit gehenden Skepsis. Wir dürfen nie vergessen, dafs wir es bei ihm nicht mit einem Geschichtschreiber, sondern mit einem Dichter zu thun haben, der mit den Thatsachen frei genug umgeht, von dem wir aber auch nicht voraussetzen können, dals er selbst immer ganz genau unterrichtet gewesen sei und sich niemals durch ein blofses Gerücht oder eine grundlose Vermuthung habe täuschen lassen. Wir dürfen jedoch ebensowenig übersehen, dafs dieser Dichter von der ihm bekannten Geschichte nicht aus blofser Willkühr, sondern aus bestịmten künstlerischen oder wissenschaftlichen Motiven abweicht. Wenn uns daher bei Platon Angaben begegnen, deren Geschichtlichkeit in Frage steht, so wird immer das erste sein müssen, dafs wir untersuchen, ob und wie weit bei ihm nach dem ganzen Zusammenhang, in dem diese Angaben vorkommen, die Absicht, uns wirkliche Geschichte zu geben, vorausgesetzt werden därf, oder ob umgekehrt in diesem Zusammenhang ein Grund zur Abweichung von der Geschichte, zur Erfindung jener Angaben lag. Haben wir uns aber auch überzengt, dafs er Geschichte geben will, so fragt es sich immer noch, ob ihm diese genau genug bekannt war, um seine Aussagen durchaus zuverlässig erscheinen zu lassen. Auf die eine wie auf die andere von diesen Fragen wird vielleicht in der Mehrzahl der Fälle keine ganz sichere Antwort möglich sein. Allein in der gleichen Lage befinden wir uns 
bei historischen und kritischen Forschungen unzähligemale: statt der Gewilsheit, die wir suchen, müssen wir uns mit einer gröfseren oder geringeren Wahrscheinlichkeit begnügen. So unerwünscht uns diefs aber auch sein mag, so steht es doch nicht in unserer Macht, es zu ändern: wir haben das unsrige gethan, wenn wir feststellen, was sich feststellen lälst, im übrigen aber der Grenzen unseres Erkennens uns bewulst sind, um da, wo es uns an einem sicheren Wissen fehlt, wenigstens nicht durch den Schein desselben getäuscht zu werden. 